\title{
The Basic Principles of PCB Wiring Layout for Switching Power
}

\author{
Chunfei Jü, ${ }^{1, a}$ Jingchao Liü, bै $^{*}$ \\ (Xijing University)
}

\author{
${ }^{1}$ Xijing University, No.1 Xijing Road, Chang'an District, Xi'an City, Shaanxi Province, China \\ ${ }^{2}$ Xijing University, No.1 Xijing Road, Chang'an District, Xi'an City, Shaanxi Province, China

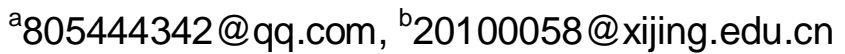

\begin{abstract}
Keywords: Switching power supply; Printed circuit board; Electromagnetic compatibility
\end{abstract}
\begin{abstract}
With the development of power electronics technology, switching power supply has been applied in various fields such as military products, scientific research equipment, automatic control, liquid crystal display, monitoring and security, digital products and instruments with its characteristics of high efficiency, low power consumption, small size and light weight. PCB is a fundamental part of switch power supply, if the size is too large, wiring impedance increases, the ability to resist noise is reduced, but if the size is too small, you need to take account into the issue of heat dissipation and components interact with each other, and the electromagnetic compatibility is critical to determine its performance index. This paper expounds the basic principles of switching power supply PCB wiring layout, to ensure its good electromagnetic compatibility, high performance of the switch power supply.
\end{abstract}

\section{Switching power supply}

At present, the problem of energy and environmental protection is increasingly prominent, electronic equipment needs to adopt more efficient and reliable power supply. In the early days, the power supply was usually the heavy linear power supply of power frequency rectification, with the highest efficiency being only 30\% 40\%. At present, this low-ripple linear power supply is only used in a few occasions, and high-performance switching power supply is widely used in other engineering applications.

With the progress of semiconductor technology, more and more advanced manufacturing technology not only improve the transient response and reliability of the power semiconductor devices, also improved the conduction loss of the device, which will greatly improve the system reliability, stability of switch power supply, electromagnetic compatibility and efficiency, and help to improve the system dynamic parameter, improve the working frequency. In recent years, with the rapid development of digital technology, digital control technology brings switching power supply into the era of intelligent control.

The power module is a kind of power supply, which can supply power for digital or analog load such as integrated circuit, microprocessor and memory.

Switching Power Supply is the Product of Modern Power Electronics Technology. It can maintain stable output voltage by controlling the time ratio of switching on or off. With the development of power electronics, switching power supply technology has been innovated. At present, the switching power supply, characterized by high efficiency, low power consumption, small size and light weight, represents the development direction of the stabilized voltage power supply, has become the mainstream product of the stabilized voltage power supply, and is also an indispensable power supply mode for the information industry with rapid development.

The Development of Switching Power Supply Has Great Significance in Environmental Protection, Resource Saving and Electronic Control Miniaturization. Switching power supply products are widely used in military products, scientific research equipment, automatic control, liquid crystal display, monitoring and security, digital products and instruments. 


\section{Printed Circuit Board}

Printed Circuit Board (PCB) design is an important part of electronic product design. The development course of electronic products is from the semiconductor radio, the black and white television develop to the current giant electronic computer, the national defense advanced science, the communication, the high precision medical equipment and so on the cutting-edge field, we clearly saw the electronic industry rapid development. The improvement of function is based on the improvement of internal electronic circuit PCB, which makes great improvement from the material selection, board design, anti-interference and other performance requirements. With the improvement of people's living standard, waste discharge treatment in the production process of PCB board is also an issue that manufacturers are increasingly concerned about. Therefore, when designing PCB, we should consider not only the product function, but also the electrical performance, manufacturing technology requirements, economy, so as to shorten the trial production cycle of new PCB.

Since the 1960s, PCB has adopted hole metallization and electroplating to solve the problem of interlayer connection or conduction. In fact, in the past, the present or the future, PCB plating copper is the core problem to solve hole connect copper plating, copper plating thickness uniformity and fill in the hole copper plating. As the high density and structure of the PCB products diversified development, PCB plating technology has made rapid progress, but the core problem of electroplating copper has no change. So far, PCB electroplating has experienced conventional dc electroplating, direct electroplating, pulsed electroplating, new dc electroplating, and the core is still the problem of "hole" conduction between PCB layers.

With the development of electronic technology, design and manufacturing automation have become the development trend of the electronic industry. The design of electronic products is more complicated, but the life cycle of its products is shorter and shorter. The design of circuit schematic diagram is the premise of PCB board production. The design of PCB board is an important link in the realization of circuit principle, and the quality of printed circuit board design will directly affect the quality of products. The following is an introduction to the layout.

\section{The Importance of Reasonable Design of Printed Boards}

When designing the PCB of switching power supply, the size of PCB is the first to consider the high quality and low cost. When the PCB size is too large, the wiring increases, impedance increases, noise resistance decreases, and the cost increases accordingly. However, if the size of PCB is too small, the problem of heat dissipation and interference of adjacent components need to be considered. Besides, the high frequency in the working process of switching power supply also makes the problem of electromagnetic interference exist. Therefore, the electromagnetic compatibility of PCB should not be neglected. The PCB layout design is one of the important factors affecting the performance of switching power supply.

Electromagnetic compatibility mainly starts from three aspects: reducing the interference signal of the interference source, cutting off the transmission channel of the interference signal and enhancing the anti-interference ability of the circuit itself. It is also should be noticed in the process of designing switch power supply that added absorption circuit, soft switch technology, EMI filter etc. The following will focus on the PCB layout design, that how to ensure its performance is the best, electromagnetic compatibility is the strongest, and the product quality.

\section{Basic Design Principles of PCB}

We design the switching power supply PCB wiring layout, generally in accordance with the schematic diagram, in line with the "first big after small, difficult before they easy" principle of layout. The core important components in the layout needs to give the priority, and should be arranged the location of the components according to the principle diagram. We should keep the signal flow in the same direction as far as possible, especially need to pay attention to the following 
questions.

The Main Loop. In switching power supply, the loop contains input filter capacitance, primary winding of high frequency transformer and switch tube constitute loop, secondary winding of high frequency transformer, rectifier or relay diode and output filter capacitor, all of which have large impulse current.

In the actual production, there are four such loops, input rectifier filter circuit, power switch circuit, output rectifier circuit and output filter circuit, which have a great impact on the conduction and radiation of EMC, as well as the stability of the circuit, output ripple and noise. The power switch circuit and output rectifier circuit are the most important. The area of these four loops must be small, and the wiring should be as short and thick as possible, so as to reduce the loss of the absorption loop and improve the power efficiency.

The Switching Devices. Switching devices are heating devices, such as the power switch tube and secondary rectifier tube, which generate more heat during the operation of the power module, especially the collector of the power switch tube and the anode of the secondary rectifier tube. When the PWM control chip works, the high temperature will lead to oscillation frequency drift and reference voltage deviation, which will affect the performance index of the switching power supply. During wiring, these devices should not be close to each other, and the copper covering area (including the lead) should be small. When wiring, no acute angle or right angle can be found. As far as possible, the wiring should be round to reduce the influence of electromagnetic emission on EMC.

The Signal Ground. The switching power common ground does not clearly distinguish between the power ground and the control ground in the schematic diagram, also known as the power ground and the signal ground. Power ground, as the common potential reference point of the large current loop, plays a very important role in the circuit. When designing the printed board, the wiring should be wide enough and copper should be coated for heat dissipation when necessary. In general, in the PCB wiring design, the power ground and the signal ground are deliberately separated, and the single point is grounded, that is, the switching power of large current and the control signal of small current are separately collected and then grounded.

Other Questions. The filter capacitance of IC should be placed between VCC and GND. When the first stage filter capacitors are placed in parallel after the secondary rectification, the lead impedance of the two capacitors should be as consistent as possible, so that the two capacitors can share the ripple current on average, and the temperature difference is small and the service life is consistent. The parallel connection of secondary filter capacitors after secondary rectification adopts the method of end beam tail to make the ripple current complete through the filter capacitor in the shortest path, ensuring small ripple and good effect.

In addition, the layout design of switching power supply PCB should be based on the principle as short as possible, and the spacing should conform to the national standard. High frequency, high voltage, large current devices and low frequency, low voltage and small signal devices should be separated as far as possible, and analog signals should be separated from digital signals.

\section{Summary}

PCB wiring layout design of the power supply module has a close relationship with the electromagnetic compatibility and output efficiency, and determines the performance of the switch power supply, so in the actual production it should be in strict accordance with the basic principles of the wiring layout. At the same time, we should accumulate experience in the work and make improvements to accomplish the components put reasonable and minimal impact between each other. The line layout that is right can guarantee to conform to the standard of high-performance switching power supply. 


\section{Reference}

[1] Lin Chen. Electromagnetic compatibility analysis and design in design of high-frequency switching power supply [J]. Information communication,2017(07):63-65.

[2] Chunya Zhang, Lin He, Zhiguo Zhang. Overview of switch power technology development [J]. Microelectronics,2016,46(02):255-260+272.

[3] Jingchao Liu, Hongzhu Cai. Basic principles of layout of PCB of switching power supply [J]. Wireless Internet technology,2015(01):108.

[4] Yapei Pan. Design and implementation of high frequency switching power supply based on DSP [D]. Nanjing university of science and technology,2013.

[5] Wenzhi He, Dongyuan Qiu, Wenxun Xiao, Bo Zhang. Thermal design of high-frequency high-power switching power supply structure [J]. Journal of electrical technology,2013,28(02):185-191+218.

[6] Huisen He. Study on electromagnetic compatibility design and stability based on ac-dc switching power supply system [D]. Xi 'an university of electronic science and technology,2012.

[7] Xiangpeng Liu. Study on electromagnetic compatibility of PCB layout of switching power supply [D]. China civil aviation university,2011.

[8] Qingjiang Hou, Liqiang Zhang, Donggang Xu. Analysis of the basic principle and development trend of switching power supply [J]. Manufacturing automation,2010,32(09):160-162+169.

[9] Cheng Wang. Study on PCB wiring of electronic products [J]. Science and technology information,2009(31):104+148.

[10] Xin Wu, Liting Qian, Minxi Pang. Research on electromagnetic compatibility of printed circuit board of switching power supply [J]. Journal of electronics and information,2001(02):181-186.

[11] Xin Wu, Liting Qian, Minxi Pang, Junming Li. Method of EMC auxiliary design of switching power supply printed circuit board [J]. Journal of electrical engineering technology,2000(04):62-66.

[12] Wu Xin, Pong M.H., Lee C.M.. Research on near-field interference of switching power sources [J]. Power electronics technology,1999(04):59-61. 